Trivent Publishing

(C) The Authors, 2016

Available online at http://trivent-publishing.eu/

Engineering and Industry Series

Volume Power Systems, Energy Markets and Renewable Energy Sources in

South-Eastern Europe

\title{
The SCADA System at the Generation Center of Hydro North
}

\author{
Siniša Sekulić, Krešimir Vrdoljak
}

Končar - Power Plant and Electric Traction Engineering Inc., Fallerovo šetalište 22 10000 Zagreb, Croatia, sinisa.sekulic@koncar-ket.hr, kresimir.vrdoljak@koncar-ket.hr

\begin{abstract}
The Generation Center North has recently been revitalized. Apart from all new hardware, the revitalized center includes a new SCADA system, the expansion of the telecommunications system and the adaptation of process control and information systems in hydro power plants (HPPS). SCADA's communication with process stations in HPPs is done using the IEC 60870-5-104 communication protocol, while communication with external centers is done using the ICCP communication protocol. The SCADA system PROZA NET is based on the newest computer technology and openness standards in order to provide the possibility of long-term upgrade and improvement, hence ensuring the necessary safety in system operation.
\end{abstract}

\section{Keywords}

SCADA; PROZA NET; control; supervision; Generation Center North 


\section{Introduction}

Three hydro power plants (HPP) are remotely controlled and monitored from the Generation Center North (GCN) in Croatia. They are HPP Varaždin, HPP C̆akovec and HPP Dubrava, all within the Drava River catchment, located in northern Croatia. Hydro North (HN) hydropower system is shown in Fig. 1.

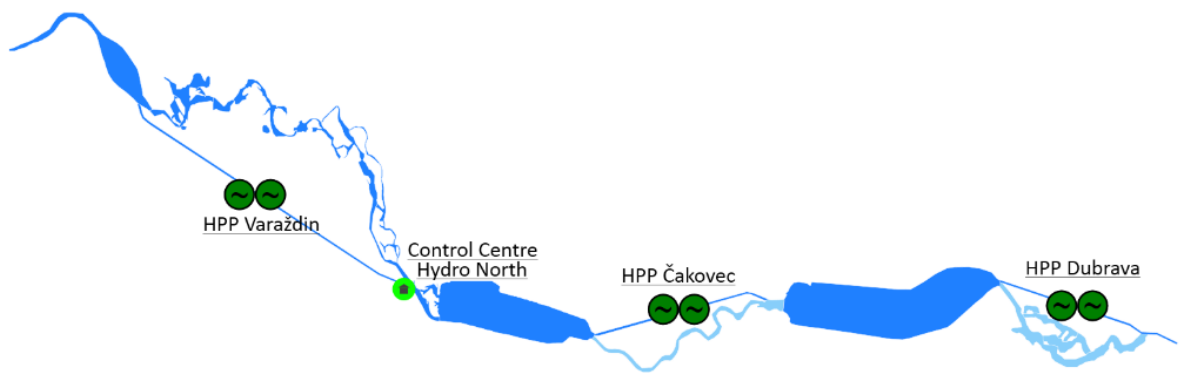

Fig. 1. The hydropower system of Hydro North

The HN hydropower system has a total installed capacity of $250 \mathrm{MW}$ with an average yearly production of $1.2 \mathrm{TWh}$. The functions of catchment supervision, production planning, operation and control are conducted from GCN.

GCN was recently revitalized [1]. Apart from all new hardware, the revitalized center now includes the following applications and functions: SCADA, production planning and short-term optimization, automatic generation scheduling, voltage and reactive power control, smart metering, database integration, data warehouse and web portal. The revitalization also comprised telecommunications system expansion and adaptation of process control and information systems in the HPPs and in the center itself.

The new SCADA system in GCN is PROZA NET. It is based on the latest computer technology and openness standards in order to provide the possibility of long-term upgrade and improvement, hence ensuring the necessary safety in system operation. The PROZA NET SCADA system utilizes an architecture designed to conform to the high demand of real-time operation as well as large system databases and WAN communication. Secure and reliable operation by redundancy of critical SCADA functions at the hardware and software level is also ensured in the revitalized GCN.

The SCADA system is based on two SQL databases with the following functions:

- real-time database (ProcessModel) contains process model definitions, lists of events and actual system states snapshot;

- historical database (Archive) is used for archiving of all relevant measured data. 


\section{System Concept}

All critical hardware and software segments of the system are redundant. The distributed system architecture is briefly described below.

\section{A. Implemented Hardware}

The logical arrangement of the most important GCN's system components is shown in Fig. 2. Core SCADA components are highlighted and they consist of workstations and servers with client applications and critical server modules. There are two SCADA servers in the redundant configuration including communication and process model handling modules. The other pair consists of two servers with database management applications. Workstations are used for the engineering, supervision, visualization and control of the processes in GCN.

Server pairs operate in the dual mode - one server is primary and the other secondary standing in the "hot standby" mode. If any primary database server function is interrupted, it is immediately replaced by the same secondary server function. Each server and workstation has two network cards (network teaming). The disk space on all the servers is in RAID array. Database management servers use the shared disk space and they are organized into a cluster.

The usage of a video wall is a part of the everyday routine. The video wall is operated by a controller that also uses the PROZA NET Client application for monitoring and control operations. A video panel at the entrance into the GCN building represents a part of the visualization equipment as well. It switches among a set of chosen SCADA displays.

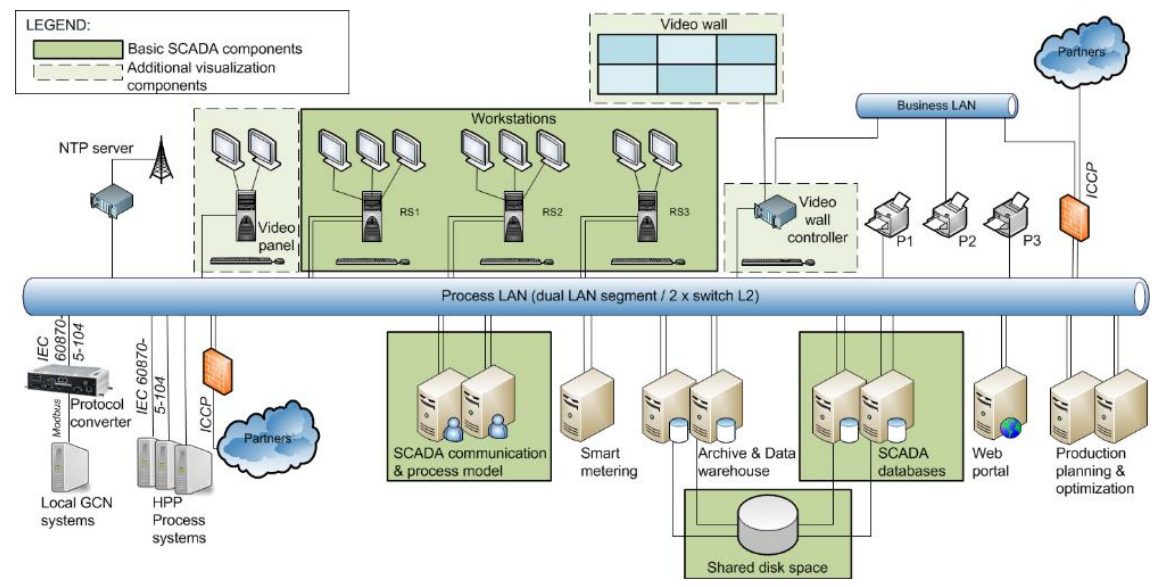

Fig. 2. SCADA system hardware 


\section{B. SCADA Software Modules}

The SCADA system software modules interact with each other and perform the following functions:

- FEP (Front-End Processor) - a communication service responsible for gathering information from process-communication nodes. The communication with process systems in GCN and hydro power plants is carried out using IEC 60870-5-104 communication protocol. Other protocols are also supported such as: IEC 60870-5-101, IEC 60870-5103, DNP 3.0, LON, IEC 61850, ModbusRTU, ModbusTCP and OPC.

- Process Server - a real-time database service and an event list server. It receives information from FEP and maps it in the model understandable and accessible to the operator. The Process Server is also responsible for processing lists of events, alarms and failures. Information is organized in columns, while time stamps are associated with records to keep the chronological order.

- Archive Server - stores data into a historical database located in the shared disk space. Information is stored according to rules defined in the archive model. Measured values are organized into groups. Each group is defined by its storage frequency, dead zone and type of archiving (spontaneous or cyclic).

- SCADA calculation server (PmBlock Server) - performs logical and arithmetic operations on real-time data.

- Process Controller - that service is responsible for implementation of user-defined algorithms that allow the enforcement of automatic generation scheduling.

Only the data archiving module (the Archive Server) resides in the "SCADA databases" server pair. All the other modules are located in the "SCADA communication and process model" server pair.

The end user (dispatcher, operator) uses the client application to monitor process information in the form of SCADA screen displays, lists of events or trend displays of measured values. The PROZA NET Client application establishes a connection with SCADA software modules. Workstations also have ability to access the video wall.

\section{User Interface Functions}

The user interface is built using Microsoft .NET WPF (Windows Presentation Foundation) technology. SCADA screen displays provide an insight into functioning of all important basin segments. HMI is used for opening of 
SCADA displays, viewing of trends and reports and performance of control operations.

\section{A. Process Visualization}

Fig. 3 shows the organization of SCADA screen display.

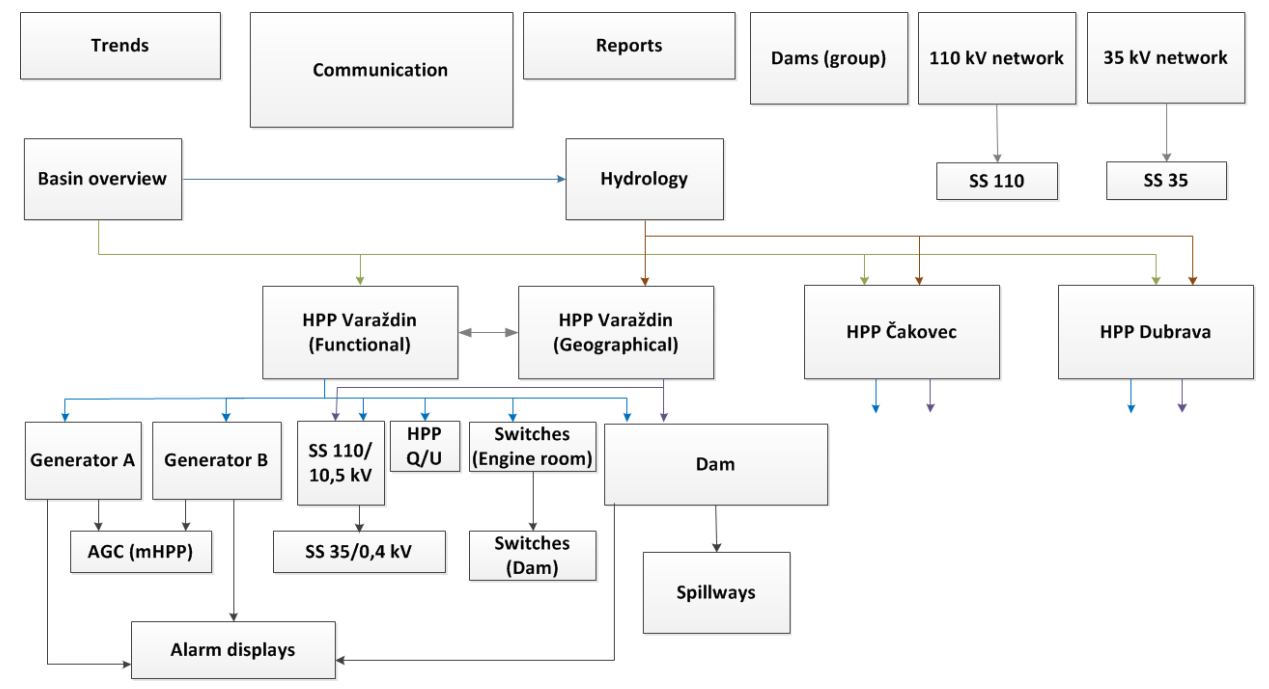

Fig. 3. SCADA displays organization

The following displays are available:

- Geographical and functional presentations of the basin, individual hydro power plants and generators;

- Switchyards (single-line diagrams);

- Dams and spillways;

- Engine room and dam control switches;

- Group and individual alarms;

- Communication status and SCADA module states;

- Trends and reports.

Hydro-mechanical objects in GCN (spillways with flap gates and segment floodgates) are animated quickly in such a way that they provide information on the current process state.

Generators and turbines are also animated along with start and stop sequences. Alarms assigned to individual facilities are grouped to unburden the most commonly used SCADA displays, but the list of events contains all individual alarms. 


\section{B. Control Mechanisms}

PROZA NET includes different control modes such as simultaneous managing of several facilities or running of a predefined control sequence. Control dialogs include automatic interlock checks. They can consist of several steps to be followed by the operator during issuing of a control order to increase the security.

There are several types of control dialogs:

- Generator start and stop commands;

- Switchgear commands (breaker and disconnector control);

- Set point commands.

Fig. 4 shows a typical breaker control dialog. The user selects the breaker and checks interlocking conditions before selecting, confirming and issuing of a command.

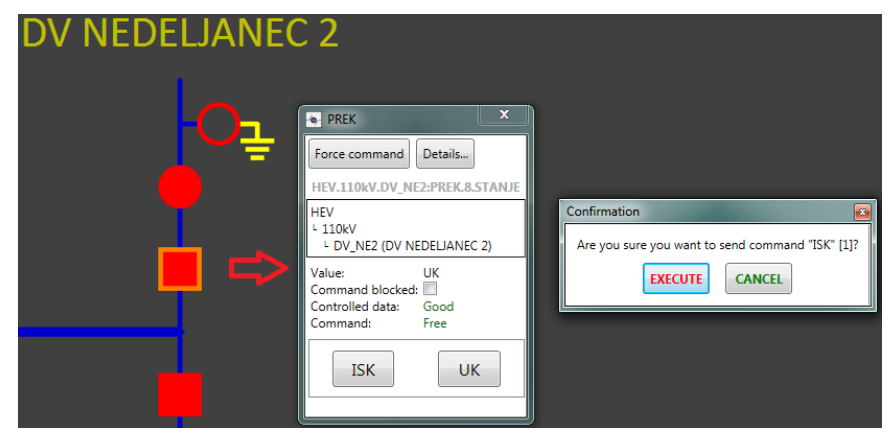

Fig. 4. Example of a breaker control dialog

Since the management of each individual facility depends on the state of physical and software switches, information about their positions are logically grouped in SCADA displays.

Each workstation offers a possibility to open several screen displays in the same monitor or in several monitors. In such a way control switches of all the facilities can be checked up before performance of any control action. Every control action can be disabled unless necessary conditions are fulfilled. An example of the stated event is shown in Fig. 5 - a dialog for managing of the generator active and reactive power is different depending on control switches (automatic scheduling plan execution and group reactive power control). 


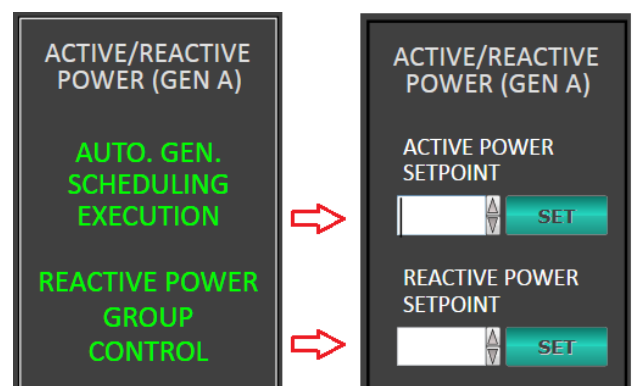

Fig. 5. Example of power set point dialog

\section{Communication and System Monitoring}

SCADA servers communicate with HPP process systems and the local GCN system (shown in Fig. 2) using IEC 60870-5-104 communication protocol. Each HPP has a main process station that acts as a data concentrator for underlying facilities and as a communicator for the center. It periodically sends a heartbeat message to the control center enabling the SCADA system to detect any communication issue and to alert the dispatching crew. The main process station also sends information on operating statuses of all underlying process stations to the control center.

The PROZA NET system gathers information from local GCN process systems including signals from different groups such as the local power supply system, hardware equipment state (servers and server components, network equipment), temperature measuring systems and system clock data. Some of those data (diesel generator, local power distribution or temperature sensors) arrive to the SCADA system indirectly using the communication-control device L-KKU (Linux based protocol converter). L-KKU communicates with subordinated devices over ModbusRTU and ModbusTCP protocols and converts information into IEC 60870-5-104 telegrams before sending them to SCADA.

All the other data are collected using the SNMP v2 and v3 protocol. A part of the SCADA screen showing data gathered by the SNMP protocol for the disk storage and switch monitoring is shown in Fig. 6.

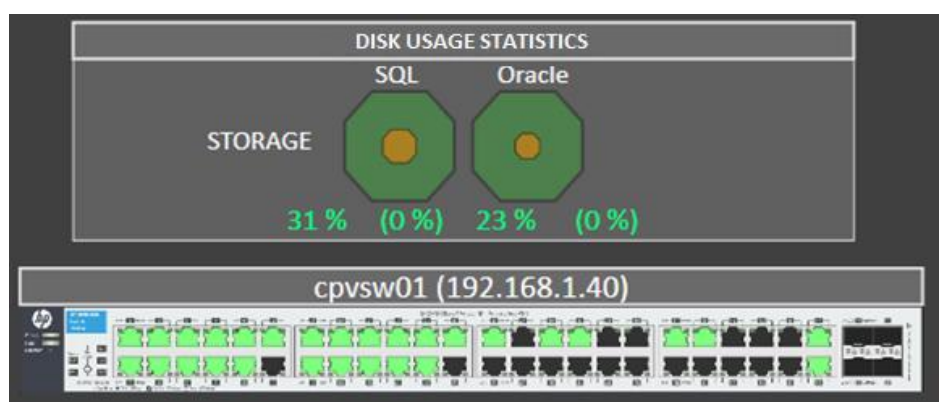

Fig. 6. SNMP disk and switch usage statistics 


\section{Reporting Functions}

SCADA archive client tools are used to define a data model for archiving of information. Measurements are grouped depending on their types (voltage, flow, water level, power, current, temperature and other) and the HPP they come from.

Each spontaneously-gathered value is treated before storage. Its dead zone, high and low limits and other constraints are checked up, and included automatically in derived calculations of:

- 1-minute averages,

- 1-minute maximums,

- 1-minute minimums.

Spontaneously collected measurements are stored in Archive Server memory for a configurable period of time to enable users to view online trend curves as shown in Fig. 7.

"Reports", one of SCADA screen displays allows the user to open semiautomatic reports in a predefined form (containing the same group of process measurements).Data in each report will be updated whenever the report is opened again or whenever the time interval is changed.

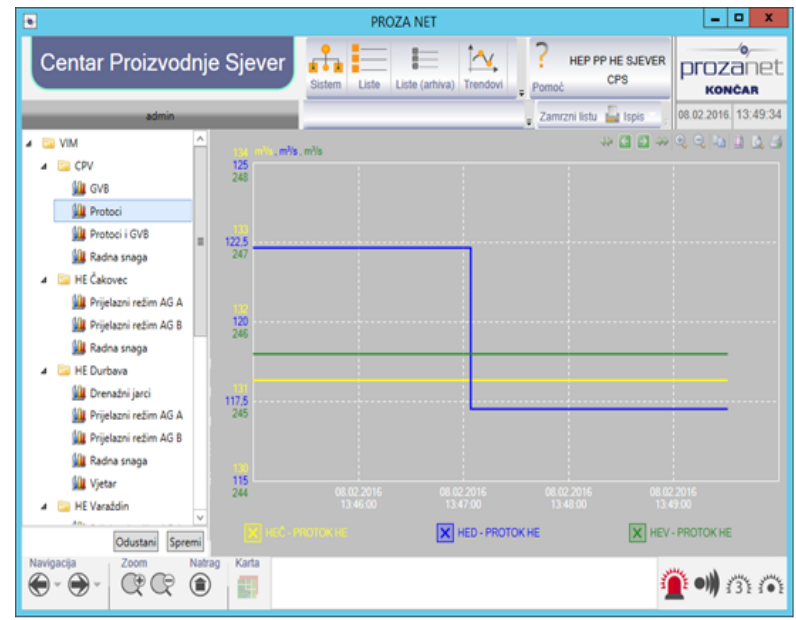

Fig. 7. Trend-curves and reports

\section{Integration with Other Systems}

The SCADA system interacts with the following components in GCN, as shown in Fig. 2:

- Archive database, data warehouse;

- Smart metering systems;

- Production planning and optimization;

- Web portal. 
The archive database (warehouse) is automatically populated by information from the SCADA historical database. Information are stored in the archive database for a longer period of time.

The dispatcher has an option to run MARS application (Management and Automated Reading) and to review states of smart metering systems. The link of MARS application is integrated into the SCADA PROZA NET user interface.

Production planning and optimization servers also send results to the SCADA system enabling the option for automatic execution of the production plan.

Information from the SCADA system are filtered and transferred to a web server where they are offered to customers residing on process and business networks through a web browser.

Two integration examples are depicted in subsections A and B.

\section{A. Automatic Execution and Monitoring of Production Plan}

The function of the Automatic Execution and Monitoring of the Production Plan is a part of the automatic generation control system and an integral SCADA component. It is also an integral part of GCN in general [2].

The function of the automatic execution of the production plan has the following inputs:

- Optimal and confirmed production schedule resulting from production planning;

- Measurements necessary for monitoring of the execution of the schedule;

- HPPs restrictions on power generation;

- Function outputs are set point commands;

- Generator(s) active power and

- HPPs flow (optional).

Commands are being sent to units that participate in the execution of the production plan. A block diagram of an automatic and a manual execution of the production plan is shown in Fig. 8.

Monitoring of the execution of the production plan is performed with the help of automatic (real-time) checking of:

- Deviation of the total basin production from the scheduled production;

- Deviation of the dam water level from the scheduled level;

- Deviation of the generator active power from the scheduled value.

The user interface of PROZA NET SCADA alarms dispatchers automatically whenever any deviation exceeds its default value. 


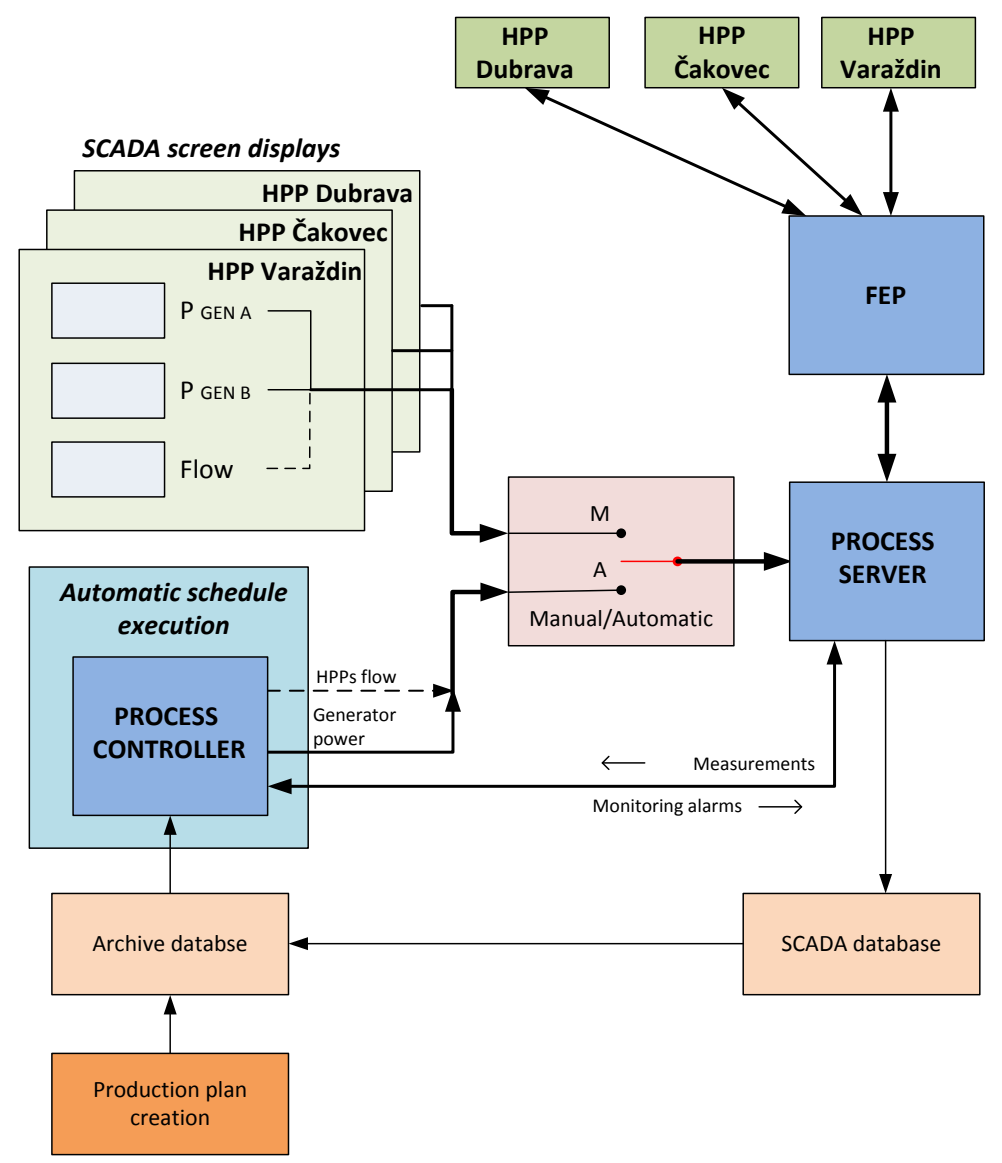

Fig. 8. Components involved in execution of scheduled plan

\section{B. Web Access}

The SCADA System PROZA NET transfers the filtered set of data to the Web Server (shown in Fig. 2) that adjusts the display format and ensures that information are consistent with the actual process situation. It also enforces system security rules (firewall configuration and user authorization) and the dispatcher safety (read-only access).

PROZA NET WebUI enables users to access the system over a standard web browser. Access to data on the current system state is provided in WebUI screen displays "Basin Overview" and "Hydrological Data". Fig. 9 shows the WebUI interface to SCADA data belonging to the "Basin Overview" screen display. 


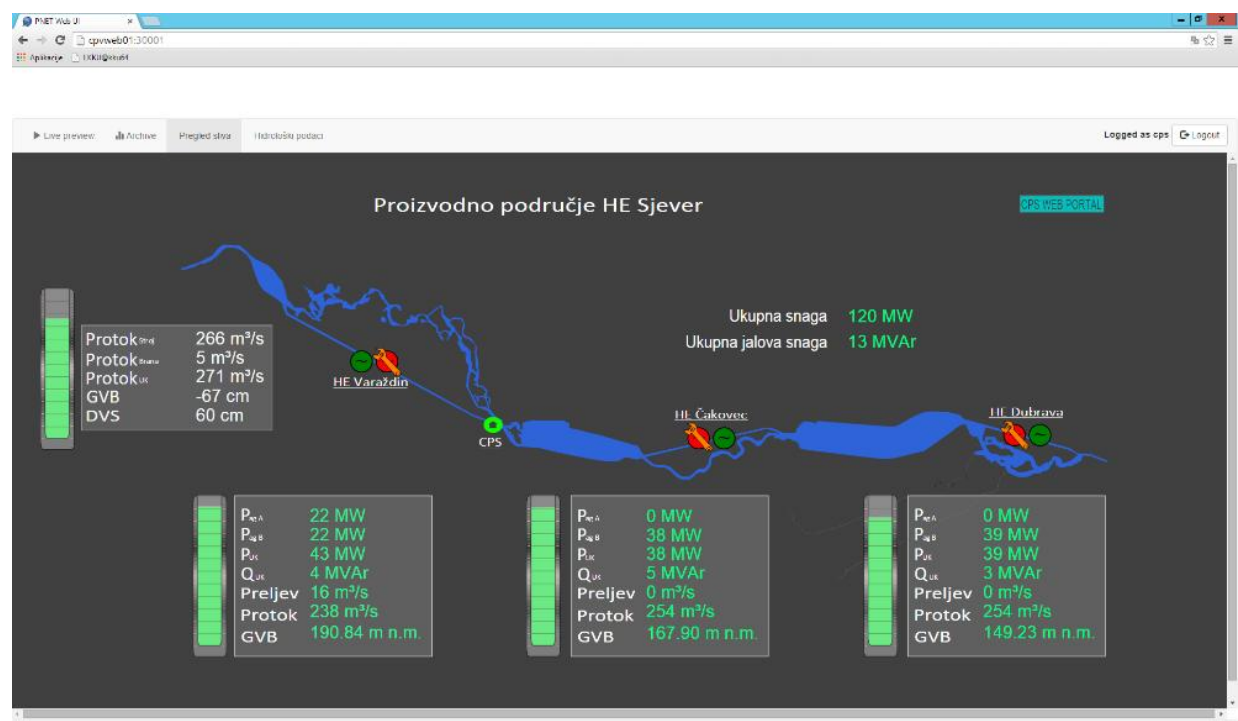

Fig. 9. "Basin Overview" web site

The web interface does not support updates of control operations or system parameters. The access control mechanism is based on roles that are valid in the system.

The web server communicates with the SCADA server process using the web socket protocol that allows refreshing of the user interface without reloading of the entire form.

\section{Generation Control Centers in Croatia}

The GCN is the second generation control center for HPPs in Croatia. HPPs placed in several basins of southern Croatia (or South Hydro Area) are monitored and their production optimized from Generation Center Dalmatia. It was constructed in 2013 [3]. The Generation Center West intended for the control of the West Hydro Area has just entered the contracting phase. All three generation centers and associated HPPs are shown in Fig. 10. 


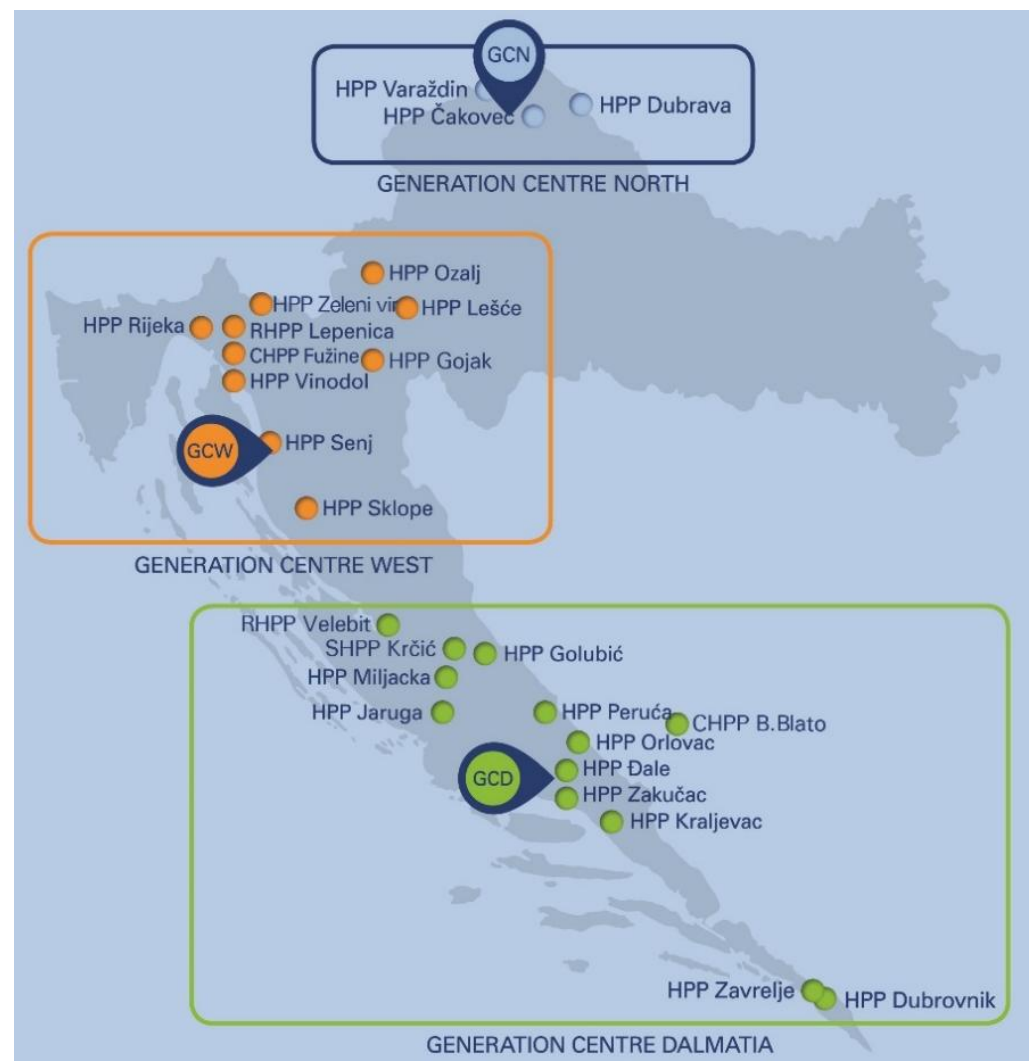

Fig. 10. Generation centers in Croatia

\section{Conclusion}

The SCADA System PROZA NET is successfully implemented to the Generation Center North in Croatia. Typical production center applications are installed as a part of the SCADA System. They are also used to integrate a number of external systems. Not only the Contractor's expertise, but also the longstanding user's experience has been exploited for that successful integration. The SCADA system is open for further upgrades in terms of expanding the number of process points and introduction of additional facilities and functions.

\section{References}

K. Vrdoljak, B. Horvat, I. Strnad, Ž. Štefan, "Razlozi i opseg revitalizacije centra daljinskog nadzora i upravljanja KL Varaždin" ["Reasons and Scope of Revitalization of Remote Control and Monitoring Center KL Varaždin“], $11^{\text {th }}$ Symposium on Power System Management, Opatija, November, 2014 (In Croatian). 
K. Vrdoljak, B. Pavlović, B. Horvat, S. Sekulić, I. Strnad, Ž. Štefan, Z. Mudri, M. Krneta, "Optimiranje rada sustava lanca hidroelektrana na PP HE Sjever" ["Optimization of Hydro Power Plant Cascade System Operation at Hydro North"], $12^{\text {th }}$ HRO CIGRÉ Session, Šibenik, November, 2015 (In Croatian).

M. Bačić, S. Sekulić, E. Vrljičak, Z. Mihaljević, L. Bitunjac, "Daljinski nadzor i upravljanje hidroelektranama iz Centra sliva rijeke Cetine" ["Hydro Power Plant Remote Monitoring and Control from the Cetina River Basin Centre"], $10^{\text {th }}$ Symposium on Power System Management, Opatija, November, 2012 (In Croatian). 